\section{Resolutividade dos serviços de saúde por inquérito domiciliar: percepção do usuário}

\author{
Case-resolving capacity of health care services \\ according to a household survey: users' perceptions
}

\author{
${ }^{1}$ Escola de Enfermagem, \\ Universidade de São Paulo, \\ Brasil. \\ 2 Faculdade de Saúde \\ Pública, Universidade de São \\ Paulo, Brasil. \\ Correspondência \\ R. N. T. Turrini \\ Departamento de \\ Enfermagem Médico \\ Cirúrgica, Escola de \\ Enfermagem, Universidade \\ de São Paulo. \\ Av. Dr. Enéas de Carvalho \\ Aguiar 419, São Paulo, SP \\ 05403-000, Brasil. \\ rturrini@usp.br
}

\begin{abstract}
The objective of this study was to identify the caseresolving capacity of health care services used by individuals who reported a health problem within two weeks prior to the interview and to unveil their respective perceptions of the solution to the problem. Data were obtained from a household morbidity survey including 10,199 interviewees, performed in the southwestern area of the city of São Paulo, Brazil, in 1989/1990. Some health problem was reported by $31.3 \%$ of interviewees, and $47.7 \%$ sought help to solve their problem. Primary health services were the main gateway into the health system (35.7\%), followed by hospitals (25.4\%), and outpatient clinics (24.3\%). The case-resolving capacity in the medical services was greater than 90\%; 44.5\% had their problem solved, $35.5 \%$ were under treatment, and $10.5 \%$ reported that their problem had not been solved. The largest proportion of problems solved belonged to respiratory and digestive system, infectious and parasitic diseases, and ill-defined symptoms, signs, and conditions. Due to the large proportion of individuals still in treatment, this study does not allow one to conclude that the health care services had a high case-resolving capacity, although they were capable of handling the patient demand.
\end{abstract}

Health Services; Outcome Assessment (Health Care); Morbidity
Ruth Natalia Teresa Turrini 1

Maria Lúcia Lebrão 2

Chester Luiz Galvão Cesar ${ }^{2}$

A resolutividade dos serviços de saúde é uma maneira de se avaliar os serviços de saúde a partir dos resultados obtidos do atendimento ao usuário. O conceito de resolutividade foi utilizado em investigação ${ }^{1}$ realizada na década de 80 para analisar as características tecnológicas do processo de trabalho na rede estadual de centros de saúde de São Paulo, e relacionava-se à resolução final dos problemas trazidos ao serviço e à satisfação dos clientes e do médico.

No modelo assistencial decorrente das ações integradas de saúde, proposto em 1983, a principal medida de efetividade era dada pela resolutividade 2 . A questão resolutividade presente nos documentos relacionava-se ao incremento de equipamentos médicos (medicamentos, exames subsidiários, aparelhagem de RX etc.) e à necessidade de implementar pronto-atendimento nos centros de saúde.

A resolutividade dos serviços no modelo hierarquizado por níveis de atenção pode ser avaliada por dois aspectos. O primeiro, dentro do próprio serviço, quanto à capacidade de atender à sua demanda e de encaminhar os casos que necessitam de atendimento mais especializado e, o segundo, dentro do sistema de saúde, que se estende desde a consulta inicial do usuário no serviço de atenção primária à saúde até a solução de seu problema em outros níveis de atenção à saúde.

Siméant 3,4 utilizou a capacidade resolutiva para avaliar os serviços na zona rural do Chile 
sob dois aspectos: um deles quantitativo, considerando que a demanda varia no tempo e no espaço, e outro qualitativo, que se altera em função da etiologia das necessidades que motivam a demanda (cirurgia, clínica médica, obstetrícia, pediatria etc.) e da complexidade destas necessidades. Esse autor refere, ainda, que há dois tipos de demanda expressa pela comunidade: uma, espontânea, que surge das necessidades percebidas, e outra, induzida, decorrente das necessidades de prevenção nem sempre sentidas pela população, mas sim impostas pelo Estado, por meio dos programas de saúde pública.

A capacidade resolutiva quantitativa depende do número e da distribuição de horas dos profissionais que se podem mobilizar para absorver a demanda onde e quando ela ocorre. É avaliada pelo número de clientes atendidos sobre o número de clientes que solicitaram o serviço, e o critério de mensuração será a taxa de demanda reprimida. $\mathrm{O}$ aspecto qualitativo da demanda depende da natureza e da complexidade dos recursos físicos, humanos, materiais, diagnósticos e terapêuticos envolvidos na prestação dos serviços. Este é avaliado pelo resultado do número de casos resolvidos sobre o número de casos atendidos, e o critério de medição será o número de clientes encaminhados. Do grau de capacidade dos recursos humanos e da tecnologia empregada nos serviços de apoio dependerá o grau de complexidade da prestação outorgada ${ }^{3}$.

Como a percepção do cliente sobre determinado serviço é particular, pois se desconhecem os critérios que o cliente utiliza para definir a qualidade do serviço, também é difícil identificar o que significa para ele um serviço ser capaz de resolver seu problema de saúde. Supõe-se que quanto maior a resolutividade de um serviço, mais voltado e preparado está para atender as necessidades de saúde da população, mesmo que isto signifique encaminhá-lo para outro serviço para continuidade do atendimento.

A resolutividade tem sido discutida com diferentes abordagens, mais ou menos abrangentes e, portanto, poder-se-ia dizer que ela depende de quem avalia e o que se avalia no momento. Reunindo-se os diferentes enfoques é possível dizer que a resolutividade envolve aspectos relativos à demanda, à satisfação do cliente, às tecnologias dos serviços de saúde, à existência de um sistema de referência preestabelecido, à acessibilidade dos serviços, à formação dos recursos humanos, às necessidades de saúde da população, à adesão ao tratamento, aos aspectos culturais e sócioeconômicos da clientela, entre outros.

Este estudo teve por objetivo identificar a resolutividade dos serviços de saúde utilizados pela população que referiu pelo menos um problema de saúde nos 15 dias anteriores à entrevista e procurou ajuda para resolvê-lo, na região sudoeste da Grande São Paulo, Brasil. A resolutividade foi analisada a partir da percepção do entrevistado sobre a solução do seu problema de saúde e da capacidade do serviço em atender a demanda segundo os relatos dos indivíduos sobre atendimento e encaminhamento.

\section{Material e métodos}

Para este estudo de abordagem descritiva utilizou-se o banco de dados do inquérito domiciliar Morbidade Referida e Utilização de Serviços de Saúde no ERSA-12, 1989/1990 (Faculdade de Saúde Pública, Universidade de São Paulo/Secretaria de Estado da Saúde de São Paulo) realizado na região sudoeste da Grande São Paulo entre julho de 1989 e 1990.

Os municípios envolvidos no estudo pertenciam ao Escritório Regional de Saúde de Itapecerica da Serra - ERSA 12 (Itapecerica da Serra, Taboão da Serra, Embu, Embu-Guaçu, Juquitiba e Cotia, incluindo Vargem Grande). Os municípios mais distantes de São Paulo apresentavam características rurais, enquanto os mais próximos eram tidos como cidades dormitório.

A amostra representativa da população residente em cada um dos municípios foi obtida pela técnica de amostragem de área 5 . Inicialmente cada município foi considerado um conjunto de setores e subsetores censitários e, posteriormente, cada grupo foi dividido em dois estratos com base na densidade domiciliar. Depois dessa estratificação, sortearam-se trinta setores ou subsetores em cada município. Numa segunda etapa, sortearam-se os quarteirões no estrato $1 \mathrm{e}$ os domicílios no estrato 2. Para o estrato 1 houve uma terceira etapa de sorteio em que se selecionaram os domicílios nos quarteirões já sorteados. O inquérito compreendeu 10.199 entrevistados e para este estudo foram utilizadas as informações daqueles que referiram algum episódio de doença nos 15 dias anteriores à entrevista (3.191). Dado que a maioria deles $(80,2 \%)$ referiu apenas um evento, para a análise considerou-se o primeiro episódio referido.

O inquérito utilizou um formulário composto de 14 blocos, e para este estudo foram incluídas as respostas dos blocos dois (sexo, idade), três (morbidade referida para os 15 dias anteriores à entrevista, problemas e procuras, tratamento), bloco 14 (condição sócio-econômica). A maioria das perguntas era do tipo fechada, com exceção dos sintomas ou diagnósticos, exames realizados e aquelas que pediam para especificar as respostas "sim", "não" ou "outro". 
As variáveis utilizadas foram sexo, idade, município de residência, escolaridade do chefe de família, classe social do chefe de família (burguesia, nova pequena burguesia, pequena burguesia tradicional, proletariado típico, proletariado não-típico e subproletariado conforme conceito de classe social operacionalizado por Lombardi et al. 6), tipo de assistência médica (Sistema Único de Saúde - SUS, privado, convênio, outros), morbidade referida (CID-9 7), quem procurou para resolver o problema de saúde (médico, outro profissional de saúde, outros), tipo de serviço procurado (hospital, unidade básica de saúde, clínica/ambulatório, pronto-socorro), tempo para buscar ajuda, localização do serviço (município de residência, ERSA-12, Município de São Paulo, outros), prescrição de medicamentos, forma de obtenção do medicamento, tratamentos seqüenciais (inalação, fisioterapia, massagem e tratamento caseiro), exames pedidos, serviços onde realizou os exames, encaminhamentos a especialistas e a outros serviços, a percepção sobre a resolução do problema (referido pelo entrevistado), como solucionou o problema não resolvido pelo atendimento recebido.

A análise dos dados foi feita utilizando-se a freqüência absoluta e a porcentagem na amostra ponderada considerada representativa da população, uma vez que a pirâmide populacional por faixa etária da região foi semelhante à pirâmide populacional da amostra ponderada. Para os dados de morbidade, utilizou-se o coeficiente de prevalência por mil habitantes. A capacidade resolutiva quantitativa foi calculada pela porcentagem de entrevistados que procuraram o atendimento e foram atendidos e, a capacidade resolutiva qualitativa, pela porcentagem de entrevistados atendidos e não encaminhados.

\section{Resultados}

A prevalência de morbidade da região do ERSA-12 foi de $265,2 \%$ o pessoas na amostra ponderada. As doenças respiratórias foram as mais referidas com um coeficiente de prevalência de $92,3 \%$, seguidas pelos sinais e sintomas mal definidos $(63,8 \%$ o)

Dos 3.191 entrevistados, 1.473 (47,7\%) mencionaram ter procurado algum tipo de ajuda para resolver o seu problema, com predomínio de mulheres (57,3\%); 64,1\% (1.020) dependiam do SUS para o atendimento em saúde, 27,7\% (309) tinham um plano de saúde ligado a empresas e 7,8\% (93) outros tipos de cobertura.

A procura de ajuda diferiu conforme o tipo de acometimento e chegou a $100 \%$ para determinados problemas de saúde, como as complicações da gravidez, parto e puerpério e algumas afecções originadas no período perinatal. Além desses, os problemas que mais motivaram a procura de serviços de saúde foram neoplasmas, doenças do aparelho geniturinário, doenças infecciosas e parasitárias (Tabela 1).

Os entrevistados que não procuraram ajuda para resolver o problema de saúde apresentaram principalmente afecções do capítulo XVI com maior referência à insuficiência respiratória, nervosismo e debilidade; do capítulo XIII com destaque para as dorsopatias e reumatismos; do capítulo VIII com elevada menção de gripes e resfriados; do capítulo IX com predomínio de úlceras e gastrites; do capítulo VI caracterizado por doenças do ouvido; e do capítulo IV salientando-se as anemias.

O principal profissional procurado para resolver o problema de saúde foi o médico $(76 \%)$ em todos os municípios. Entre os entrevistados que buscaram um profissional não-médico (11,2\%), 98,5\% deles se dirigiram ao farmacêutico.

O serviço de atenção primária à saúde foi a principal porta de entrada no sistema $(35,7 \%)$, seguido pelos hospitais $(25,4 \%)$ e clínicas/ambulatórios (24,3\%) (Tabela 2).

Os serviços utilizados para o atendimento localizaram-se principalmente no próprio município de residência $(54,8 \%)$ e 35,5\% dos respondentes utilizaram serviços na cidade de São Paulo. A maioria dos entrevistados foi atendida no próprio serviço procurado (93,5\%). Com relação à capacidade resolutiva qualitativa, o número de encaminhamentos para especialistas ou outros serviços foi considerado pequeno $(2,7 \%)$, assim como aqueles encaminhados para internação após a consulta (1,6\%). A capacidade resolutiva quantitativa dos serviços utilizados pelos entrevistados foi de $97,8 \%$.

Observou-se que tanto a capacidade resolutiva quantitativa quanto a qualitativa diferiu em função do tipo de serviço utilizado. Embora o número de entrevistados que procuraram os serviços de saúde oferecidos pelas empresas tenha sido pequeno, em relação aos demais serviços utilizados pela população da região, a resolutividade deste tipo de serviço foi de $100 \%$. Em seguida, as clínicas e ambulatórios foram as mais resolutivas, o que poderia ser atribuído à especificidade destes serviços.

O maior número de encaminhamentos foi feito para os hospitais $(55,4 \%)$, depois para os ambulatórios/clínicas (20,1\%), para as unidades básicas de saúde $(14,8 \%)$ e os prontos-socorros $(9,7 \%)$. Assim como ocorre na contra-referência, os hospitais também remeteram considerável número de pessoas $(43,4 \%)$ às unidades básicas de saúde. A maioria dos clientes foi en- 
Distribuição de moradores segundo o problema referido por capítulos do CID-9 7 e procura de ajuda para solucioná-lo. Região sudoeste da Grande São Paulo, Brasil, 1989/1990.

\begin{tabular}{|c|c|c|c|c|c|c|}
\hline \multirow[t]{3}{*}{ Problema de saúde } & \multicolumn{4}{|c|}{ Procura de ajuda } & \multicolumn{2}{|c|}{ Total } \\
\hline & \multicolumn{2}{|c|}{ Sim } & \multicolumn{2}{|c|}{ Não } & \multirow[b]{2}{*}{$\mathrm{n}$} & \multirow[b]{2}{*}{$\%$} \\
\hline & $\mathbf{n}$ & $\%$ & $\mathrm{n}$ & $\%$ & & \\
\hline I - Doenças infecciosas e parasitárias & 79 & 67,7 & 40 & 32,3 & 119 & 100,0 \\
\hline II - Neoplasmas & 2 & 84,3 & 1 & 15,7 & 3 & 100,0 \\
\hline III - Doenças das glândulas endócrinas, nutrição, metabolismo e transtornos imunitários & 11 & 61,4 & 5 & 38,6 & 16 & 100,0 \\
\hline IV - Doenças de sangue e órgãos hematopoiéticos & 7 & 50,5 & 8 & 49,5 & 15 & 100,0 \\
\hline V-Transtornos mentais & 1 & 63,9 & 4 & 36,1 & 5 & 100,0 \\
\hline VI - Doenças do sistema nervoso e dos órgãos do sentido & 68 & 49,6 & 55 & 50,4 & 123 & 100,0 \\
\hline VII - Doenças do aparelho circulatório & 120 & 64,3 & 78 & 35,7 & 198 & 100,0 \\
\hline VIII - Doenças do aparelho respiratório & 502 & 45,6 & 517 & 54,4 & 1019 & 100,0 \\
\hline IX - Doenças do aparelho digestivo & 92 & 47,4 & 126 & 52,6 & 218 & 100,0 \\
\hline X-Doenças do aparelho geniturinário & 78 & 70,1 & 45 & 29,9 & 123 & 100,0 \\
\hline XI - Complicações da gravidez, do parto e do puerpério & 2 & 100,0 & - & - & 2 & 100,0 \\
\hline XII - Doenças de pele e do tecido celular subcutâneo & 40 & 66,7 & 33 & 33,3 & 73 & 100,0 \\
\hline XIII - Doenças do sistema osteomuscular e do tecido conjuntivo & 116 & 41,7 & 185 & 58,3 & 301 & 100,0 \\
\hline XIV - Anomalias congênitas & - & - & - & - & - & 100,0 \\
\hline$X V$ - Algumas afecções originadas no período perinatal & 4 & 100,0 & - & - & 4 & 100,0 \\
\hline $\mathrm{XVI}$ - Sintomas, sinais e afecções mal definidas & 314 & 39,9 & 472 & 61,1 & 786 & 100,0 \\
\hline XVII - Lesões e envenenamentos & 37 & 55,9 & 31 & 44,1 & 68 & 100,0 \\
\hline Total & 1.473 & 47,7 & 1.600 & 52,3 & 3.073 & 100,0 \\
\hline
\end{tabular}

n: número absoluto na amostra não ponderada; \%: porcentagem na amostra ponderada.

caminhada para serviços localizados no Município de São Paulo (72,1\%). Apenas 16,1\% foram encaminhados para outros serviços do próprio município e $11,3 \%$ para serviços na própria região do ERSA 12.

Analisando-se a morbidade referida dos casos encaminhados, verificou-se que a maioria pertencia às doenças dos aparelhos respiratório e digestivo. As unidades básicas de saúde foram os únicos serviços que encaminharam casos de afecções relativas ao sistema nervoso e órgãos dos sentidos, além do maior número de doenças respiratórias. Os clientes que procuraram um hospital e, posteriormente, foram encaminhados a outros serviços, foram os que mais referiram afecções classificadas como sinais e sintomas mal definidos.

A maioria dos clientes entrevistados teve medicamentos prescritos em decorrência do atendimento recebido $(85,7 \%)$. Poucos obtiveram os medicamentos gratuitamente $(29,1 \%)$, muitos os compraram (62,9\%) e alguns não os conseguiram (3,9\%).

Em 21\% (39) dos casos atendidos foi indicado tratamento seqüencial, ou seja, tratamentos complementares à terapêutica medicamentosa $\mathrm{e}$ que incluíram basicamente fisioterapia $(54,5 \%)$, inalação $(22,1 \%)$ e outros $(23,4 \%)$. Foi prescrita inalação a $1,4 \%$ (9) das pneumonias referidas e este procedimento só foi indicado nas unidades básicas de saúde.

A fisioterapia foi indicada a 23\% (12) dos entrevistados que referiram dorsopatias ou reumatismos, e os serviços em que foi prescrito este tipo de tratamento eram clínicas/ambulatórios. O tratamento caseiro também foi pouco prescrito, ou seja, para menos de $0,1 \%$ dos entrevistados. Quanto aos locais onde esses tratamentos foram realizados, $57,4 \%$ (22) foram no próprio serviço do atendimento, 22,5\% (7) em locais fora da área de estudo, com exceção de um, e 20,1\% (3) não foram realizados.

Para complementar a avaliação clínica, exames foram solicitados a $25,1 \%$ (281) dos entrevistados, num total de 430 exames distribuídos da seguinte maneira: $28,3 \%$ de sangue, $20,9 \%$ de fezes, $18,7 \%$ de urina, $15,4 \%$ de radiografias, $5 \%$ de eletrocardiogramas e 11,7\% outros tipos de exames, sendo que estes incluíram alguns procedimentos como biópsias e broncoscopias. As radiografias foram solicitadas a 6,2\% (23) dos entrevistados que referiram doença do aparelho 
Distribuição de moradores que procuraram ajuda para resolver seu problema de saúde, dentre os que referiram problema, segundo o serviço utilizado e município de residência. Região sudoeste da Grande São Paulo, Brasil, 1989/1990.

\begin{tabular}{|c|c|c|c|c|c|c|c|c|c|c|c|c|c|c|}
\hline \multirow[t]{3}{*}{ Município } & \multicolumn{14}{|c|}{ Tipo de serviço de saúde } \\
\hline & \multicolumn{2}{|c|}{$\begin{array}{c}\text { Unidades básicas } \\
\text { de saúde }\end{array}$} & \multicolumn{2}{|c|}{ Hospital } & \multicolumn{2}{|c|}{$\begin{array}{c}\text { Clínica/ } \\
\text { Ambulatório }\end{array}$} & \multicolumn{2}{|c|}{$\begin{array}{l}\text { Pronto- } \\
\text { socorro }\end{array}$} & \multicolumn{2}{|c|}{ Empresa } & \multicolumn{2}{|c|}{ Outros } & \multicolumn{2}{|c|}{ Total } \\
\hline & $\mathbf{n}$ & $\%$ & $\mathbf{n}$ & $\%$ & $\mathbf{n}$ & $\%$ & $\mathbf{n}$ & $\%$ & $\mathbf{n}$ & $\%$ & $\mathrm{n}$ & $\%$ & $\mathbf{n}$ & $\%$ \\
\hline Taboão da Serra & 50 & 36,0 & 34 & 28,8 & 34 & 32,0 & 2 & 1,4 & 1 & 0,3 & 5 & 1,5 & 126 & 100,0 \\
\hline Embu & 90 & 36,7 & 54 & 23,9 & 33 & 17,4 & 34 & 16,1 & 5 & 3,4 & 4 & 2,5 & 220 & 100,0 \\
\hline Itapecerica da Serra & 59 & 26,1 & 51 & 25,2 & 55 & 22,4 & 39 & 19,2 & 4 & 3,4 & 6 & 3,7 & 214 & 100,0 \\
\hline Embu-Guaçu & 146 & 67,4 & 11 & 5,4 & 33 & 20,6 & 1 & 1,0 & 3 & 2,3 & 4 & 3,3 & 198 & 100,0 \\
\hline Juquitiba & 84 & 35,6 & 140 & 56,7 & 17 & 7,0 & 2 & 0,7 & - & - & - & - & 243 & 100,0 \\
\hline Cotia & 61 & 31,6 & 38 & 23,1 & 59 & 31,6 & 16 & 7,7 & 6 & 5,1 & 5 & 0,9 & 185 & 100,0 \\
\hline Total & 490 & 35,7 & 328 & 25,4 & 231 & 24,3 & 94 & 9,7 & 19 & 2,8 & 24 & 2,1 & 1186 & 100,0 \\
\hline
\end{tabular}

n: número absoluto na amostra não ponderada; \%: porcentagem na amostra ponderada.

respiratório. Os eletrocardiogramas foram pedidos a $5,7 \%$ (9) das pessoas que referiram afecções do aparelho circulatório e a 3,3\% (7) dos que mencionaram sinal ou sintoma mal definido relacionado a este sistema. Verificou-se que alguns exames foram mais solicitados quando o atendimento foi realizado em clínicas/ambulatórios, como a ultra-sonografia, a endoscopia e o exame de Papanicolau.

Os exames foram realizados, principalmente, no próprio município de residência $(42,6 \%)$ e em São Paulo (48,6\%). Os principais tipos de serviços utilizados para a realização dos exames foram as unidades básicas de saúde $(38,1 \%)$, os hospitais $(29,7 \%)$, as clínicas/ambulatórios (26\%) e os laboratórios $(8,9 \%)$.

\section{Percepção da solução dos problemas de saúde pelo entrevistado}

Entre os entrevistados, observou-se que 44,5\% (527) referiram solução do problema de saúde e $35,5 \%$ (544) ainda estavam em tratamento. Apenas 1,2\% (15) das pessoas foi encaminhada a um especialista e 12,3\% (213) não tiveram seu problema resolvido. A solução de problemas de saúde entre moradores que procuraram recursos no próprio município de residência foi de 41,1\% (247) (Tabela 3).

A Tabela 3 apresenta a distribuição da solução dos problemas referida segundo variáveis de morbidade e de utilização dos serviços. A solução de problemas foi similar para o tipo de serviço utilizado, com exceção das clínicas/ambulatórios e para os problemas atendidos no município de residência ou do ERSA-12. No entanto, com relação à não solução do problema, os serviços menos resolutivos foram os hospitais e prontossocorros com mais de $10 \%$ de casos não solucionados.

A maior menção de não resolução do problema foi observada entre os que não tiveram medicamentos prescritos. Para a forma de obtenção de medicamentos, os resultados da solução do problema variaram pouco entre as categorias, porém, observou-se que, para quem não obteve o medicamento ou o conseguiu de outra forma que não a explicitada, 22,8\% deles não tiveram seu problema de saúde solucionado.

Quando o tempo para buscar ajuda para resolver o problema de saúde foi superior a seis dias, houve maior porcentagem de casos não solucionados (20\%).

A maior proporção de problemas resolvidos esteve entre as doenças respiratórias, as infecciosas e os sinais e sintomas mal definidos. Considerando-se a proporção em cada capítulo do CID-9 (Tabela 4), a maior freqüência de casos não resolvidos foi observada nas doenças do sistema osteomuscular e do tecido conjuntivo, basicamente outros transtornos do dorso e não especificados e outros transtornos dos tecidos moles. O segundo grupo de doenças menos resolvidas pertencia ao sistema nervoso e aos órgãos dos sentidos, principalmente afecções do olho e ouvido que necessitavam de um especialista. O terceiro grupo, também com mais de $15 \%$ dos casos não resolvidos, foi o das doenças do aparelho digestivo, prioritariamente outras doenças e afecções dos dentes e de suas estruturas de sustentação e transtornos da função gástrica. 
Distribuição da população que procurou ajuda para resolver seu problema de saúde, segundo a resolução do problema e variáveis de utilização dos serviços. Região sudoeste da Grande São Paulo, Brasil, 1989/1990.

\begin{tabular}{|c|c|c|c|c|c|c|c|c|c|c|c|c|c|c|}
\hline \multirow[t]{3}{*}{ Variável } & \multicolumn{14}{|c|}{ Resolução do problema } \\
\hline & \multicolumn{2}{|c|}{ Sim } & \multicolumn{2}{|c|}{$\begin{array}{c}\text { Em } \\
\text { tratamento }\end{array}$} & \multicolumn{2}{|c|}{$\begin{array}{c}\text { Sim, } \\
\text { parcialmente }\end{array}$} & \multicolumn{2}{|c|}{$\begin{array}{c}\text { Não, foi } \\
\text { encaminhado }\end{array}$} & \multicolumn{2}{|c|}{ Não } & \multicolumn{2}{|c|}{ NS-NR } & \multicolumn{2}{|c|}{ Total } \\
\hline & $\mathrm{n}$ & $\%$ & $\mathrm{n}$ & $\%$ & $\mathbf{n}$ & $\%$ & $\mathrm{n}$ & $\%$ & $\mathrm{n}$ & $\%$ & $\mathrm{n}$ & $\%$ & $\mathrm{n}$ & $\%$ \\
\hline \multicolumn{15}{|l|}{ Localização do serviço } \\
\hline Próprio município de residência & 247 & 41,1 & 300 & 39,8 & 59 & 7,8 & 9 & 2,0 & 100 & 9,1 & 5 & 0,2 & 720 & 100,0 \\
\hline ERSA-12 & 40 & 49,6 & 31 & 28,1 & 4 & 4,6 & - & - & 16 & 17,7 & - & - & 91 & 100,0 \\
\hline Outros municípios & 102 & 37,6 & 146 & 46,6 & 15 & 4,0 & 6 & 1,3 & 41 & 10,0 & 2 & 0,5 & 312 & 100,0 \\
\hline \multicolumn{15}{|l|}{ Tipo de serviço } \\
\hline Unidade básica de saúde & 168 & 43,2 & 198 & 40,9 & 33 & 5,3 & 6 & 1,0 & 63 & 9,6 & 1 & 0,0 & 469 & 100,0 \\
\hline Hospital & 98 & 44,1 & 120 & 39,9 & 22 & 2,2 & 4 & 2,1 & 49 & 11,1 & 3 & 0,6 & 296 & 100,0 \\
\hline Clínica/Ambulatório & 79 & 36,2 & 115 & 43,8 & 17 & 9,5 & 4 & 1,1 & 27 & 9,3 & 2 & 0,1 & 244 & 100,0 \\
\hline Outros (inclusive pronto-socorro) & 44 & 41,9 & 44 & 33,2 & 6 & 9,0 & - & - & 18 & 15,7 & 1 & 0,2 & 113 & 100,0 \\
\hline \multicolumn{15}{|l|}{ Pedido de exames } \\
\hline Sim & 60 & 28,8 & 151 & 54,2 & 23 & 4,0 & 3 & 0,3 & 44 & 12,7 & - & - & 281 & 100,0 \\
\hline Não & 328 & 44,7 & 327 & 36,6 & 54 & 6,6 & 12 & 2,0 & 113 & 9,8 & 6 & 0,3 & 840 & 100,0 \\
\hline \multicolumn{15}{|l|}{ Prescrição de medicamentos } \\
\hline Sim & 347 & 42,3 & 439 & 43,2 & 62 & 4,8 & 7 & 0,9 & 118 & 8,5 & 7 & 0,3 & 980 & 100,0 \\
\hline Não & 42 & 32,9 & 36 & 24,6 & 16 & 13,9 & 8 & 5,4 & 39 & 23,2 & - & - & 141 & 100,0 \\
\hline \multicolumn{15}{|l|}{ Forma de obtenção do medicamento } \\
\hline Gratuito & 118 & 45,6 & 145 & 39,9 & 18 & 5,1 & 4 & 1,4 & 34 & 8,0 & 2 & 0,0 & 321 & 100,0 \\
\hline Compra & 204 & 40,8 & 272 & 46,0 & 43 & 5,3 & 3 & 0,8 & 63 & 7,0 & 2 & 0,1 & 584 & 100,0 \\
\hline Outros & 23 & 41,9 & 20 & 32,8 & 1 & 0,3 & - & - & 21 & 22,8 & 3 & 2,2 & 68 & 100,0 \\
\hline \multicolumn{15}{|l|}{ Tempo para procurar o serviço (dias) } \\
\hline Até 2 & 342 & 50,5 & 279 & 30,5 & 54 & 6,4 & 4 & 0,6 & 115 & 10,9 & 8 & 1,1 & 82 & 100,0 \\
\hline De 3-5 & 101 & 45,3 & 102 & 37,3 & 17 & 5,8 & 5 & 2,5 & 37 & 9,1 & 1 & 0,0 & 263 & 100,0 \\
\hline 6 ou + & 71 & 26,3 & 140 & 47,7 & 22 & 4,0 & 6 & 2,0 & 56 & 20,0 & - & - & 295 & 100,0 \\
\hline
\end{tabular}

NS-NR: não sabe/não respondeu; n: número absoluto na amostra não ponderada; \%: porcentagem na amostra ponderada.

Os moradores dos municípios mais próximos à capital foram aqueles que mais tiveram seu problema de saúde resolvido. Para as pessoas cujos chefes de família tinham maior escolaridade, observou-se maior porcentagem de problemas resolvidos, principalmente entre os de ensino superior; e entre os analfabetos a maior freqüência de casos não resolvidos. A freqüência de solução dos casos, segundo o sexo, foi semelhante para ambos. Em relação à faixa etária, observou-se maior proporção de não solução de problemas de saúde entre os entrevistados com idade superior a 29 anos. O tipo de assistência médica utilizado não mostrou variações quanto à solução dos problemas de saúde, mas entre aqueles que utilizaram o SUS, houve maior menção de não solução do problema $(14,8 \%)$ (Tabela 5).

Dentre os indivíduos que referiram ter tido seu problema de saúde parcialmente resolvido, apenas 5\% (4) procuraram outro tipo de ajuda para resolvê-lo. Entre aqueles que disseram que o problema não foi resolvido, 8,8\% (17) procuraram outro tipo de ajuda. Àqueles que referiram não ter procurado outro tipo de ajuda, perguntou-se como conseguiram solucionar seu problema de saúde e observou-se que 84,5\% (255) das pessoas continuavam com a queixa, 3,3\% (15) delas se automedicaram, 3,8\% (11) fizeram um tratamento caseiro e 1,3\% (2) evoluiu para a cura espontânea.

Aos entrevistados que não procuraram ajuda para resolver o problema de saúde $(52,3 \%)$ lhes foi perguntado como o solucionaram. A maioria continuava com o problema de saúde $(38,5 \%)$ ou se automedicou (32,3\%), 15,9\% fizeram um tratamento caseiro, $10,1 \%$ evoluíram para cura espontânea e 3,1\% outros. 
Distribuição da população que procurou ajuda para resolver seu problema de saúde, segundo a resolução do problema e a morbidade referida (capítulos do (ID-97). Região sudoeste da Grande São Paulo, Brasil, 1989/1990.

\begin{tabular}{|c|c|c|c|c|c|c|c|c|c|c|c|c|c|c|}
\hline \multirow[t]{3}{*}{ Variável/Afecção referida } & \multicolumn{14}{|c|}{ Resolução do problema } \\
\hline & \multicolumn{2}{|c|}{ Sim } & \multicolumn{2}{|c|}{$\begin{array}{c}\text { Em } \\
\text { tratamento }\end{array}$} & \multicolumn{2}{|c|}{$\begin{array}{c}\text { Sim, } \\
\text { parcialmente }\end{array}$} & \multicolumn{2}{|c|}{$\begin{array}{c}\text { Não, foi } \\
\text { encaminhado }\end{array}$} & \multicolumn{2}{|c|}{ Não } & \multicolumn{2}{|c|}{ NS-NR } & \multicolumn{2}{|c|}{ Total } \\
\hline & $\mathbf{n}$ & $\%$ & $\mathbf{n}$ & $\%$ & $\mathbf{n}$ & $\%$ & $\mathbf{n}$ & $\%$ & $\mathbf{n}$ & $\%$ & $\mathbf{n}$ & $\%$ & $\mathbf{n}$ & $\%$ \\
\hline 1. Doença infecciosas e parasitárias & 45 & 62,2 & 22 & 22,6 & 5 & 5,5 & - & - & 5 & 7,2 & 1 & 2,6 & 78 & 100,0 \\
\hline 2. Neoplasmas & - & - & - & - & - & - & 1 & 100,0 & - & - & - & - & 1 & 100,0 \\
\hline \multicolumn{15}{|l|}{ 3. Doença de glândulas endócrinas, nutrição e } \\
\hline metabolismo, e transtornos imunitários & 2 & 38,9 & 4 & 38,2 & 1 & 7,9 & - & - & 3 & 15,0 & - & - & 10 & 100,0 \\
\hline 4. Doença de sangue e órgãos hematopoiéticos & 3 & 36,4 & 3 & 59,2 & - & - & - & - & 1 & 4,4 & - & - & 7 & 100,0 \\
\hline 6. Sistema nervoso e órgãos dos sentidos & 25 & 29,9 & 28 & 48,2 & 2 & 1,4 & 1 & 0,8 & 10 & 19,7 & - & - & 66 & 100,0 \\
\hline 7. Doença do aparelho circulatório & 22 & 25,2 & 61 & 58,7 & 9 & 2,6 & 1 & 0,3 & 16 & 11,5 & 1 & 1,7 & 110 & 100,0 \\
\hline 8. Doença do aparelho respiratório & 218 & 50,6 & 169 & 31,3 & 43 & 8,3 & 1 & 0,6 & 51 & 8,2 & 5 & 1,0 & 487 & 100,0 \\
\hline 9. Doença do aparelho digestivo & 29 & 38,7 & 33 & 35,3 & 3 & 4,9 & 3 & 3,7 & 19 & 17,2 & - & - & 87 & 100,0 \\
\hline 10. Doença do aparelho geniturinário & 21 & 33,1 & 31 & 53,0 & 2 & 0,4 & - & - & 16 & 13,5 & - & - & 70 & 100,0 \\
\hline 11. Complicações da gravidez, parto e puerpério & - & - & - & - & 1 & 100,0 & - & - & - & - & - & - & 1 & 100,0 \\
\hline 12. Doença de pele e do tecido celular subcutâneo & 14 & 33,5 & 13 & 48,3 & 2 & 2,5 & - & - & 8 & 13,5 & - & - & 37 & 100,0 \\
\hline \multicolumn{15}{|l|}{ 13. Doença do sistema osteomuscular e do } \\
\hline tecido conjuntivo & 15 & 16,3 & 48 & 35,7 & 12 & 13,3 & 2 & 2,9 & 34 & 31,2 & 2 & 0,6 & 113 & 100,0 \\
\hline 15. Algumas afecções do período perinatal & 1 & 100,0 & - & - & - & - & - & - & - & - & - & - & 1 & 100,0 \\
\hline 16. Sintomas, sinais e afecções mal definidas & 119 & 50,0 & 118 & 32,3 & 18 & 2,8 & 4 & 1,7 & 46 & 13,0 & 1 & 0,2 & 306 & 100,0 \\
\hline 17. Lesões e envenenamento & 13 & 62,2 & 14 & 26,4 & 1 & 5,7 & 2 & 1,9 & 4 & 3,8 & - & - & 34 & 100,0 \\
\hline Total & 527 & 44,6 & 544 & 35,5 & 99 & 5,8 & 15 & 1,1 & 213 & 12,3 & 10 & 0,7 & 1.408 & 100,0 \\
\hline
\end{tabular}

NS-NR: não sabe/não respondeu; n: número absoluto na amostra não ponderada; \%: porcentagem na amostra ponderada.

\section{Discussão}

A maior freqüência de afecções respiratórias também foi observada em outros estudos, tanto em áreas urbanas quanto rurais $3,4,8,9$. A procura de ajuda para a solução de problemas manteve-se abaixo dos $50 \%$ como evidenciado em outro estudo ${ }^{10}$ e atribuída ao elevado número de problemas, cuja gravidade é tão pequena que não chega a gerar demanda de serviços e a resolução ocorre por automedicação ou com recursos de pessoas mais experientes da comunidade. A análise por capítulos do CID mostrou que a totalidade dos casos de complicações da gravidez, parto e puerpério procurou ajuda, o que poderia ser resultado do programa de saúde materna implantado nos serviços de atenção primária à saúde.

Questões de acesso ao serviço de saúde, como número de serviços, capacidade de atender a demanda, condições financeiras de pagar o atendimento, necessidade de faltar ao trabalho, além do modo como o indivíduo percebe a doença, também podem interferir na decisão para a procura de atendimento médico. Mas, uma vez reconhecida a necessidade de ajuda, o que fez a pessoa optar pelo farmacêutico ou pelo amigo/ vizinho é desconhecido, não podendo nem mesmo ser atribuído, neste estudo, exclusivamente à escolaridade dos chefes de família, dado que César 11 relata, em sua análise deste mesmo inquérito, que a menor procura pelo médico esteve entre os universitários.

O tipo de serviço disponível em cada município contribuiu na determinação do perfil dos estabelecimentos de saúde procurados, pois as unidades básicas de saúde foram mais procuradas nos municípios de residência, enquanto os hospitais o foram na capital. A distribuição dos entrevistados segundo o tipo de serviço utilizado foi próxima da observada na Pesquisa Nacional por Amostra de Domicílios de 1998 (PNAD-98) 12.

Embora não se saiba a respeito da proximidade da residência ao serviço de saúde, este não parece ter sido o fator principal da escolha do serviço, pois há linhas intermunicipais freqüentes para a capital, além da presença de rodovias que cruzam alguns municípios da região e facilitam o acesso à cidade de São Paulo. Como os municípios mais vizinhos a São Paulo são cidades-dor- 
Distribuição da população que procurou ajuda para resolver seu problema de saúde, segundo a resolução do problema e variáveis sócio-demográficas. Região sudoeste da Grande São Paulo, Brasil, 1989/1990.

\begin{tabular}{|c|c|c|c|c|c|c|c|c|c|c|c|c|c|c|}
\hline \multirow[t]{3}{*}{ Variável } & \multicolumn{14}{|c|}{ Resolução do problema } \\
\hline & \multicolumn{2}{|c|}{ Sim } & \multicolumn{2}{|c|}{$\begin{array}{c}\text { Em } \\
\text { tratamento }\end{array}$} & \multicolumn{2}{|c|}{$\begin{array}{l}\text { Sim, } \\
\text { parcialmente }\end{array}$} & \multicolumn{2}{|c|}{$\begin{array}{c}\text { Não, foi } \\
\text { encaminhado }\end{array}$} & \multicolumn{2}{|c|}{ Não } & \multicolumn{2}{|c|}{ NS-NR } & \multicolumn{2}{|c|}{ Total } \\
\hline & n & $\%$ & $\mathbf{n}$ & $\%$ & n & $\%$ & $\mathbf{n}$ & $\%$ & n & $\%$ & $\mathbf{n}$ & $\%$ & n & $\%$ \\
\hline \multicolumn{15}{|l|}{ Município de residência } \\
\hline Taboão da Serra & 61 & 54,8 & 57 & 36,3 & 11 & 6,1 & 3 & 2,3 & 11 & 0,5 & - & - & 143 & 100,0 \\
\hline Embu & 70 & 35,6 & 104 & 40,6 & 17 & 7,0 & 3 & 0,9 & 35 & 13,9 & 4 & 2,0 & 233 & 100,0 \\
\hline Itapecerica da Serra & 100 & 48,8 & 92 & 31,4 & 3 & 1,7 & 1 & 0,8 & 41 & 16,5 & 2 & 0,8 & 239 & 100,0 \\
\hline Embu-Guaçu & 71 & 39,9 & 92 & 40,0 & 18 & 6,3 & 3 & 0,6 & 34 & 13,8 & 2 & 0,3 & 220 & 100,0 \\
\hline Juquitiba & 110 & 38,6 & 106 & 33,1 & 32 & 9,5 & 2 & 0,6 & 51 & 17,7 & 2 & 0,5 & 303 & 100,0 \\
\hline Cotia & 115 & 47,9 & 93 & 31,6 & 18 & 6,0 & 3 & 0,8 & 41 & 13,7 & - & - & 270 & 100,0 \\
\hline \multicolumn{15}{|l|}{ Escolaridade } \\
\hline Analfabeto & 50 & 46,7 & 53 & 19,7 & 13 & 13,0 & - & 1,9 & 25 & 20,2 & 2 & 0,4 & 143 & 100,0 \\
\hline Ensino fundamental & 384 & 43,6 & 415 & 37,1 & 70 & 5,2 & 14 & 1,4 & 165 & 12,0 & 8 & 0,7 & 1056 & 100,0 \\
\hline Ensino médio & 49 & 54,1 & 37 & 30,6 & 11 & 6,0 & - & - & 10 & 9,3 & - & - & 107 & 100,0 \\
\hline Ensino superior & 26 & 60,9 & 8 & 19,3 & 5 & 17,0 & 1 & 0,8 & 3 & 2,0 & - & - & 43 & 100,0 \\
\hline \multicolumn{15}{|l|}{ Faixa etária (anos) } \\
\hline 1 dia a 9 & 226 & 51,4 & 179 & 33,4 & 32 & 4,6 & 1 & 0,6 & 59 & 8,5 & 4 & 1,5 & 501 & 100,0 \\
\hline $10-29$ & 141 & 48,9 & 92 & 32,4 & 15 & 7,2 & 2 & 0,4 & 39 & 11,1 & - & - & 289 & 100,0 \\
\hline $30-49$ & 73 & 37,3 & 80 & 37,8 & 17 & 4,8 & 4 & 2,7 & 43 & 17,1 & 1 & 0,3 & 218 & 100,0 \\
\hline $50-69$ & 67 & 22,0 & 160 & 49,1 & 27 & 7,9 & 8 & 2,5 & 57 & 17,9 & 3 & 0,6 & 322 & 100,0 \\
\hline $70-79$ & 20 & 30,1 & 33 & 40,8 & 8 & 8,0 & - & - & 15 & 19,2 & 2 & 1,9 & 78 & 100,0 \\
\hline \multicolumn{15}{|l|}{ Sexo } \\
\hline Masculino & 254 & 47,6 & 233 & 34,3 & 44 & 6,0 & 7 & 1,0 & 81 & 10,3 & 3 & 0,8 & 622 & 100,0 \\
\hline Feminino & 273 & 42,1 & 311 & 36,5 & 55 & 5,7 & 8 & 1,3 & 132 & 13,9 & 7 & 0,5 & 786 & 100,0 \\
\hline \multicolumn{15}{|l|}{ Classe social } \\
\hline Subproletariado & 46 & 49,1 & 54 & 35,4 & 7 & 4,3 & 3 & 0,4 & 20 & 10,5 & 1 & 0,3 & 131 & 100,0 \\
\hline Proletariado típico & 172 & 47,7 & 135 & 24,7 & 30 & 5,5 & 2 & 0,6 & 78 & 19,8 & 4 & 1,7 & 421 & 100,0 \\
\hline Proletariado não-típico & 150 & 39,1 & 210 & 42,9 & 32 & 5,0 & 7 & 2,5 & 71 & 10,2 & 2 & 0,3 & 472 & 100,0 \\
\hline Pequena burguesia tradicional & 92 & 54,0 & 69 & 29,3 & 21 & 6,8 & 2 & 0,4 & 23 & 9,5 & 1 & 0,0 & 208 & 100,0 \\
\hline Nova pequena burguesia & 30 & 63,3 & 7 & 16,8 & 4 & 16,8 & 1 & 0,8 & 4 & 2,3 & - & - & 46 & 100,0 \\
\hline Burguesia & 4 & 54,8 & 4 & 42,7 & 1 & 2,5 & - & - & - & - & - & - & 9 & 100,0 \\
\hline \multicolumn{15}{|l|}{ Tipo de assistência médica } \\
\hline Convênio de empresa & 114 & 44,1 & 121 & 36,5 & 23 & 9,2 & 5 & 1,5 & 38 & 8,7 & - & - & 301 & 100,0 \\
\hline Plano de saúde privado & 23 & 45,8 & 27 & 48,2 & 3 & 1,0 & - & - & 3 & 5,0 & - & - & 56 & 100,0 \\
\hline Outros convênios & 11 & 53,6 & 14 & 27,9 & 3 & 2,8 & 1 & 9,4 & 3 & 6,3 & - & - & 32 & 100,0 \\
\hline SUS & 365 & 45,5 & 355 & 32,5 & 69 & 5,3 & 9 & 0,8 & 161 & 14,8 & 8 & 1,1 & 967 & 100,0 \\
\hline
\end{tabular}

NS-NR: não sabe/não respondeu; n: número absoluto na amostra não ponderada; \%: porcentagem na amostra ponderada; SUS: Sistema Único de Saúde.

mitório, a população destes municípios pode ter optado pelos serviços da capital, justamente por serem próximos ao local de trabalho.

Segundo a Organização Mundial da Saúde, um serviço que é porta de entrada do sistema de saúde deveria ser capaz de resolver pelo menos $80 \%$ dos problemas de saúde 14 . Os serviços utilizados pelos entrevistados parecem atingir a meta, embora nem todos fossem um serviço de atenção primária à saúde e nem estivessem localizados no município de residência do entrevistado. Não foi investigado o critério para a escolha de tais serviços, mas os diferentes serviços de saúde utilizados sugerem que os entrevistados sabiam para onde se dirigir para terem seu problema de saúde solucionado.

Cabe ressaltar que os dados encontrados neste estudo resultaram das informações dos respondentes que buscaram ajuda para resolver os seus problemas de saúde, portanto trata-se da 
resolutividade percebida. Como pouco mais de um terço das pessoas que não procuraram ajuda continuava com o problema de saúde, se tivessem sido incluídas perguntas no formulário para a avaliação dos motivos pelos quais os entrevistados não procuraram ajuda, provavelmente terse-ia uma melhor avaliação da resolutividade dos serviços de saúde percebida pelos usuários.

$\mathrm{O}$ volume de encaminhamentos, neste inquérito, foi menor que o encontrado por outros autores 4,10,13. O maior encaminhamento dos hospitais para as unidades básicas de saúde evidenciou a baixa complexidade dos problemas de saúde atendidos e a tentativa destes serviços em adotar o sistema de regionalização e de hierarquização da atenção à saúde. A maioria dos clientes foi encaminhada para serviços localizados no Município de São Paulo, o que denota a dificuldade dos serviços locais em referir os clientes para serviços de maior complexidade na própria região, por ausência dos mesmos ou por falta de serviços conveniados com o SUS.

Como a população da região do ERSA-12 utilizou tanto serviços públicos quanto privados e de diferentes graus de complexidade, não se conseguiu estabelecer com base no banco de dados um fluxo hierarquizado e regionalizado no sistema de saúde da região que mostrasse integração entre as unidades. Sabe-se que por ocasião do estudo não havia para os serviços da região um sistema de referência e contra-referência estabelecido.

Neste inquérito, a distribuição de morbidade por tipo de serviço utilizado foi bastante heterogênea para todos os serviços, não tendo sido possível atribuir a utilização dos serviços a determinadas afecções.

A elevada prescrição de medicamentos esteve de acordo com outros estudos que revelam elevado índice de prescrição de medicamentos resultante da consulta médica 8,10,13,14. A obtenção dos medicamentos gratuitamente nos serviços de saúde, descrita nos diferentes estudos 10,15,16, reflete a organização dos serviços e os recursos disponíveis para fornecê-los à população. Tal situação traz implicações na qualidade e resolutividade do atendimento, no que diz respeito à oferta de medicamentos à clientela atendida que, por sua condição sócio-econômica, pode não ter como arcar com os custos da compra. Ainda, segundo os autores, a longo prazo, o não fornecimento de medicamentos pode provocar insatisfação tardia, o que levará o paciente a considerar o seu problema não resolvido e o incentivará a procurar outra unidade.

A fisioterapia para dorsopatias foi recomendada a pacientes atendidos em clínicas/ambulatórios. Essa é a forma de intervenção conser- vadora mais comum nos casos de dorsopatias 17 . Como muitos desses problemas de coluna decorrem, geralmente, da postura física adotada pela pessoa na atividade que desenvolve e do estresse da vida cotidiana, além da fisioterapia outras terapias complementares seriam de valia para o alívio dessa sintomatologia, além de favorecerem a solução do problema e melhorarem a qualidade de vida do indivíduo. Estudo de revisão sistemática observou que ensaios clínicos para avaliar a massagem como um tratamento para a dor nas costas aguda e crônica, evidenciaram efeitos positivos principalmente sob o aspecto funcional 18 .

Considerando-se que mais de $35 \%$ das afecções referidas eram doenças do aparelho respiratório, principalmente pneumonias e gripes, parece que houve pouca indicação de tratamento seqüencial, em particular a inalação. A orientação sobre a inalação caseira para as mães das crianças com afecções respiratórias é prática comum entre os profissionais de saúde que, no entanto, apesar da simplicidade não foi mencionada pelos entrevistados. As orientações sobre dietas também não foram citadas, embora se saiba que são abordagens importantes, principalmente nos casos de problemas relacionados aos aparelhos digestivo e circulatório. Esses resultados sugerem que os profissionais dos serviços locais não prescrevem tratamentos seqüenciais por motivos não investigados neste inquérito.

O percentual de pedidos de exames complementares neste inquérito foi superior ao do estudo de Halal et al. 13 em Pelotas (Rio Grande do Sul), e mais baixo se comparado aos de Madureira et al. 19 em Paulínia (São Paulo) e Campos 10 em Janaúba e Bocaiúva (Minas Gerais). As características demográficas da clientela atendida e a estrutura organizacional dos estabelecimentos de saúde também poderiam justificar as diferenças encontradas.

Exames específicos foram pouco mencionados, apesar de doenças crônico-degenerativas terem sido referidas. A não solicitação de exames que envolvem elevada tecnologia deve estar relacionada à falta de um serviço público de referência que pudesse atender à demanda e ao poder aquisitivo dos usuários. Poucos entrevistados tinham convênio de saúde privado e, além disto, exames sofisticados não eram freqüentemente cobertos por este sistema. A pergunta aberta também pode ter contribuído para que os entrevistados mencionassem apenas os exames mais comuns.

A não solução de problemas referidos neste inquérito foi menor do que a observada na Encuesta Nacional de Salud II no México, em 1994 20. Dependendo do estudo que se toma por re- 
ferência, a freqüência de solução dos problemas de saúde pode ser superior ${ }^{9}$ ou inferior 15 ao de outras investigações. As diferenças podem ser atribuídas ao tempo de ocorrência dos episódios mórbidos, restritos a 15 dias neste estudo, e ao fato de que muitos moradores do ERSA-12 procuraram serviços do Município de São Paulo, que possuem uma infra-estrutura melhor que os da região investigada.

Como a proporção de entrevistados que referiu ainda estar em tratamento foi maior entre aqueles que buscaram um serviço fora da região de estudo, é possível que possuíssem afecção que já sabiam que não poderia ser solucionada nos serviços de sua cidade. Essa conclusão se coaduna com o fato de o maior número de casos não resolvidos ter ocorrido entre aqueles que procuraram um serviço de saúde em outras cidades. No entanto, como a entrevista abordou os problemas de saúde dos 15 dias anteriores, pode não ter havido tempo para que os sinais e sintomas desaparecessem, independente do tipo de afecção e de sua complexidade.

Além dos comentários feitos sobre as limitações do questionário para determinadas análises sobre a utilização dos serviços, algumas questões ainda permanecem inconclusivas como os motivos pelos quais os entrevistados não buscaram ajuda para resolver seus problemas, principalmente entre aqueles que ainda persistem com os sintomas, ou buscaram serviços se saúde em outras regiões ou porque optaram pelo hospital e não pela unidade básica de saúde. Na investigação sobre os tratamentos seqüenciais, embora a pergunta aberta pareça a mais adequada, o indivíduo pode não entender as orientações sobre dieta ou atividade física, por exemplo, como um tratamento seqüencial.

\section{Conclusão}

A capacidade resolutiva, tanto qualitativa quanto quantitativa, dos serviços de saúde utilizados para consulta pela população da área estudada a partir das informações dos entrevistados, ultrapassou os $90 \%$, o que é significativo. Esse resultado sugere que os entrevistados tinham conhecimento de como resolver o seu problema no sistema de saúde. No entanto, isso não significa que a região de estudo fosse dotada de uma infra-estrutura capaz de atender às necessidades em saúde dos moradores, pois estes se deslocaram para diferentes municípios em busca de ajuda.

A diversidade dos serviços procurados pelos usuários, relacionada tanto à complexidade quanto à localização dos mesmos, aponta para a necessidade de uma análise cuidadosa dos serviços da região do ERSA-12, bem como da estruturação de um sistema de referência e contra-referência que faça a integração dos serviços locais com outros de maior nível de complexidade, de modo a suprir as necessidades em saúde da população.

Embora os dados da capacidade resolutiva dos serviços tenham mostrado que os serviços procurados são capazes de atender à demanda, do ponto de vista da solução de problemas relatada pelos entrevistados não se pode afirmar que os serviços eram resolutivos à época do inquérito, dada a proporção de indivíduos ainda em tratamento. Para se estudar a solução do problema relatada pelo entrevistado em investigações futuras será preciso refletir sobre quais questões seriam de interesse para abordar os aspectos relacionados à categoria "continua em tratamento".

Fato concreto foram os problemas mencionados como não solucionados e a elevada freqüência de pessoas que continuavam com a queixa. Uma análise específica dos dados relativos a esses entrevistados poderia trazer informações importantes sobre os serviços de saúde e os motivos pelos quais os usuários deixam de buscar outro tipo de ajuda quando o problema não foi solucionado.

Os dados foram analisados dez anos após a coleta, e muitas informações sobre a organização dos serviços na época não puderam ser recuperadas para melhor contextualização dos achados. No entanto, empiricamente pelos relatos dos usuários na prática profissional observa-se que a realidade retratada não está tão distante da atual. 


\section{Resumo}

Estudo para identificar a capacidade resolutiva dos serviços de saúde utilizados pela população que referiu pelo menos um problema de saúde nos 15 dias anteriores à entrevista, e conhecer sua percepção sobre a resolução deste problema. As informações foram obtidas de inquérito domiciliar realizado com 10.199 entrevistados na região sudoeste da Grande São Paulo, Brasil, entre 1989/1990. Destes, 31,3\% referiram algum episódio de doença e $47,7 \%$ procuraram ajuda para resolver o problema. O serviço de atenção primária à saúde foi a principal porta de entrada no sistema (35,7\%), seguido pelos hospitais $(25,4 \%)$ e clínicas/ambulatórios (24,3\%). A capacidade resolutiva dos serviços para as consultas médicas foi superior a 90\%; 44,5\% referiram solução do problema de saúde, 35,5\% estavam em tratamento e 10,5\% não tiveram seu problema resolvido. A maioria dos problemas resolvidos pertencia ao capítulo das doenças dos aparelhos respiratório e digestivo, infecciosas e parasitárias e sinais e sintomas mal definidos. A proporção de indivíduos ainda em tratamento não permitiu concluir que os serviços eram resolutivos à época do inquérito, embora tenham sido capazes de atender à demanda.

Serviços de Saúde; Avaliação de Resultados (Cuidados de Saúde); Morbidade

\section{Colaboradores}

R. N. T. Turrini elaborou o projeto, analisou os resultados e redigiu o manuscrito. M. L. Lebrão participou da análise de dados e coordenou a discussão dos resultados. C. L. G. Cesar participou da elaboração do instrumento e delineamento do estudo, acompanhou a coleta e análise dos dados e colaborou na discussão.

\section{Referências}

1. Gonçalves RBM. Tecnologia e organização social das práticas de saúde: características tecnológicas do processo de trabalho na rede estadual de centros de saúde de São Paulo. São Paulo: Editora Hucitec/Rio de Janeiro: ABRASCO; 1994.

2. Nemes MBI. Ação programática em saúde: recuperação histórica de uma política de programação. In: Scraiber LB, organizador. Programação em saúde. São Paulo: Editora Hucitec; 1993. p. 65-115.

3. Siméant S. Capacidad resolutiva de la demanda de atención de morbilidad a nivel primario, Chile, 1981. Bol Oficina Sanit Panam 1984; 97:125-41.

4. Siméant S. Estudio de la capacidad resolutiva de la demanda en atención de morbilidad a nivel primario (área urbana). Cuad Med Soc 1983; 24:156-69.

5. César CLG, Figueiredo GM, Westphal MF, Cardoso MRA, Costa MZA, Gattás VL. Morbidade referida e utilização de serviços em localidades urbanas: metodologia. Rev Saúde Pública 1996; 30:153-60.
6. Lombardi C, Bronfman M, Facchini LA, Victoria GG, Barros FC, Béria JU, et al. Operacionalização do conceito de classe social em estudos epidemiológicos. Rev Saúde Pública 1988; 22:253-65.

7 Organização Mundial da Saúde. Manual da classificação estatística internacional de doenças, lesões e causas de óbitos - 9a conferência de revisão. São Paulo: Centro Brasileiro de Classificação de Doenças em Português; 1985.

8. Tanaka OY, Resenburg CP. Análise da utilização pela clientela de uma unidade ambulatorial da Secretaria da Saúde do Município de São Paulo, SP (Brasil). Rev Saúde Pública 1990; 24:60-8.

9. Lebrão ML, Carandina L, Magaldi C. Análise das condições de saúde e de vida da população urbana de Botucatu, São Paulo (Brasil). IV- Morbidade referida em entrevistas domiciliárias, 1983-1984. Rev Saúde Pública 1991; 25:452-60. 
10. Campos FE. Resolutividade: uma aproximação à avaliação qualitativa dos serviços de saúde. [Tese de Doutorado]. Rio de Janeiro: Escola Nacional de Saúde Pública, Fundação Oswaldo Cruz; 1988.

11. Cesar CLG. Morbidade referida e utilização de serviços de saúde na grande São Paulo: o perfil da desigualdade em saúde [Tese de Livre-Docência]. São Paulo: Faculdade de Saúde Pública, Universidade de São Paulo; 1997.

12. Pinheiro RS, Viacava F, Travassos C, Brito AS. Gênero, morbidade, acesso e utilização de serviços de saúde no Brasil. Ciênc Saúde Coletiva 2002; 7:687-707.

13. Halal IS, Sparrenberger F, Bertoni AM, Ciacomet C, Seibel CE, Lahude FM, et al. Avaliação da qualidade de assistência primária à saúde em localidade urbana da região sul do Brasil. Rev Saúde Pública 1994; 28:131-6.

14. Leyvia-Flores R, Erviti-Erice J, Kageyama-Escobar ML, Arredondo A. Prescriptión, acesso y gasto en medicamentos entre usuarios de servicios de salud en Mexico. Salud Pública Méx 1998; 40:24-31.
15. Sousa AI. A satisfação da população com os serviços locais de saúde - Município de Itacarambi - MG [Tese de Doutorado]. Rio de Janeiro: Escola Nacional de Saúde Pública, Fundação Oswaldo Cruz; 1996.

16. Lemme AC, Noronha G, Resende JB. A satisfação do usuário em hospital universitário. Rev Saúde Pública 1991; 25:41-6.

17. Golby LJ, Moore AP, Doust J, Trew ME. A randomized controlled trial investigating the efficiency of muskulosketeletal physiotherapy on chronic low back disorders. Spine 2006; 31:1083-93.

18. Cherkin DC, Sherman KJ, Deyo RA, Shekelle PG. A review of the evidence for the effectiveness, safety, and cost of acupuncture, massage therapy and spinal manipulation for back pain. Ann Intern Med 2003; 138:871-81.

19. Madureira PR, De Capitani EM, Campos GWS. Avaliação da qualidade da atenção à saúde na rede básica. Cad Saúde Pública 1989; 5:45-59.

20. Ramírez-Sánchez TJ, Nájera-Aguilar P, NigendaLópez G. Percepción de la calidad de la atención de los servicios de salud en México: perspectiva de los usuarios. Salud Pública Méx 1998; 40:3-12.

Recebido em 21/Set/2006

Versão final reapresentada em 28/Ago/2007

Aprovado em 20/Set/2007 